\title{
Harmless Acute Pancreatitis Score
}

\author{
Bharti SV ${ }^{1}$, Sharma $A^{1}$
}

\begin{abstract}
Introduction: Acute Pancreatitis is a common disease in our region. It can range from mild to severe disease with high mortality rate. It is critical to identify patients who are at high risk for a severe disease course, since they require close monitoring and immediate aggressive treatment. Aims: To compare the effectiveness of Harmless Acute Pancreatitis Score with Ranson's scoring system in predicting the severity of Acute Pancreatitis. Methods: A prospective cross sectional study was done among 45 patients who were admitted in surgery department over a period of one year with diagnosis of acute pancreatitis. If haematocrit was less than $39 \%$ in female and less than $43 \%$ in male, serum creatinine less than two miligram /deciliter and no sign of peritonitis, it was assigned as Harmless Acute Pancreatitis Score Zero. If at least one parameter was abnormal it was assigned as Harmless Acute Pancreatitis Score +. Severe pancreatitis (poor prognosis) was considered in those who required Intensive Care Unit care, who had in hospital mortality and who had hospitalization of more than five days. Patients with on admission Ranson's score of more than three were suspected to have severe Pancreatitis. Results: There were total 45 patients, 18 females and 27 males. Twenty four patients were assigned as Harmless Acute Pancreatitis Score zero and 21 patients were assigned as Harmless Acute Pancreatitis Score +. Harmless Acute Pancreatitis Score was able to predict correctly in 18 out of 26 patients who fulfilled the criteria of poor prognosis $(\mathrm{p}<0.001)$. Conclusion: Harmless Acute Pancreatitis Score proved to be a better screening tool compared to on admission Ranson's scoring system to predict the severity of Acute Pancreatitis, which may help predict the prognosis of the patient.
\end{abstract}

Keywords: Harmless Acute Pancreatitis Score, Ranson's score, Severe Pancreatitis

\section{Authors:}

1. Dr. Shiv Vansh Bharti

2. Dr. Anup Sharma

${ }^{1}$ Department of Surgery, Nepalgunj Medical College and Teaching Hospital, Kohalpur, Banke

\section{Address for Correspondence:}

\author{
Dr. Shiv Vansh Bharti \\ Assistant Professor \\ Department of Surgery \\ Nepalgunj Medical College and Teaching Hospital \\ Kohalpur, Banke \\ Email: shivbharti26984@gmail.com
}

\section{INTRODUCTION}

Acute pancreatitis (AP) is one of the most common diseases of the gastrointestinal tract that requires urgent hospitalization and despite the special care is still associated with significant morbidity and mortality worldwide. ${ }^{1}$ Even after the advances in the treatment of severe AP the hospital mortality rate remains high. ${ }^{2,3}$ The average mortality rate approaches $2-10 \% .{ }^{4} \mathrm{~A}$ number of predictive scoring systems have been developed with the aim of assisting the clinicians in predicting prognosis during the early phase. The Ranson's and the Modified Glasgow Score contain data not routinely collected at the time of hospitalization. ${ }^{5,6}$ In addition, both require 48 hours to be completed, missing a potentially valuable early therapeutic window. ${ }^{7}$

The most commonly utilized predictive scoring system for clinical research studies in AP is the Acute Physiology and Chronic Health Examination (APACHE) II. ${ }^{8}$ However, the
APACHE II was originally developed as an intensive care tool and requires the collection of a large number of parameters, some of which may not be relevant to prognosis in AP. ${ }^{9}$

The recently developed new scoring systems such as the Bedside Index of Severity in Acute Pancreatitis (BISAP) and the Harmless Acute Pancreatitis Score (HAPS) involve a simplified approach that can be performed during the first 24 hours of hospitalization. The HAPS can predict a non-severe disease course with $96-97 \%$ specificity with a positive predictive value of $98 \% .^{10}$

\section{METHODS}

This was a hospital based cross-sectional prospective study conducted among 45 patients, who presented with first episode of acute pancreatitis. These patients were admitted in the surgery department of Nepalgunj Medical College and Teaching Hospital, Kohalpur. The study was conducted over a period of one year (February 2020 to January 2021). Ethical 
approval was taken from Institutional Review Committee (IRC).

All patients over 18 years with primary diagnosis of first episode of AP, presenting within 48 hours of onset of symptoms were included and patients with comorbidities like hypertension, diabetes mellitus, heart disease, chronic kidney disease, chronic liver disease and patients with recurrent pancreatitis or chronic pancreatitis were excluded.

Data including age, sex and etiological factors were recorded. Serum hematocrit and serum creatinine levels were recorded which was sent on admission in Emergency Department (ED) and signs of peritonitis were recorded after evaluation by the surgical team. Informed consent was taken and HAPS was recorded on admission. If all three parameters were normal; haematocrit less than $39 \%$ in females and less than $43 \%$ in males, serum creatinine less than two miligram/deciliter and no signs of peritonitis, it was assigned as HAPS 0 and was predicted to follow the non-severe course. If at least one parameter was abnormal it was assigned as HAPS +. Similarly, these patients were also evaluated on the basis of Ranson's criteria on admission and divided into Ranson's low risk and high risk categories.

Patients were categorized as Ranson's low risk if on admission Ranson's score was less than three and high risk, if on admission score was more than three. Patients with Ranson's score of more than three were predicted to have severe AP. Severe pancreatitis (poor prognosis) was considered in those who required ICU care, who had in-hospital mortality and/or who had hospitalization for more than five days. Data were processed and analyzed by using Statistical Package for Social Science (SPSS) software version 25.P value less than 0.05 was considered statistically significant.

\section{RESULTS}

This study enrolled 45 patients who were diagnosed with first episode of AP. The mean age was 42.23 years. There were 27 male patients and 18 female patients enrolled. The most common etiology was biliary, followed by alcohol and less common etiology was idiopathic. (Figure 1)

HAPS and on admission Ranson's score was calculated for all the patients. There were $24(53 \%)$ patients in the HAPS-0 group and the remaining $21(46 \%)$ patients had at least one parameter positive who were grouped as HAPS+. According to the Ranson's score, $37(82 \%)$ patients were classified as low risk ( $<3$ points) and $8(17 \%)$ were classified as high risk ( $>3$ points).

In our study, serum haematocrit was high in $3(6.6 \%)$ of total 45 patients. Serum creatinine was more than two miligram/ deciliter in nine patients (20\%).Signs of peritonitis were present in nine out of 45 patients $(20 \%)$ as well. Also, there were 8 patients $(26.6 \%)$ having both peritonitis and creatinine level more than two milligram/deciliter. Three patients $(22.2 \%)$ fulfilled all the three parameters for HAPS+.

Out of twenty one patients who were HAPS + , eight of them had on admission Ranson's score more than three while 13 patients had on admission Ranson's score less than three. Eight patients who were categorized as HAPS + and Ranson's score more than three had poor prognosis. However, 10 patients with HAPS+ but Ranson's score less than three also had poor prognosis. The sensitivity of HAPS was $85.71 \%$ while specificity was $66.7 \%$. (Figure 2) (Table I and II)

Among the 24 patients with HAPS 0 and Ranson's score less than three (low risk), eight patients had poor prognosis of which five patients had to be admitted in the hospital for more than five days and three patients required ICU admission. The remaining 16 patients were admitted for less than five days in the general ward and eventually discharged. However, there were no patients who were HAPS 0 with Ranson's score more than three.(Figure 2)(Table I) Similarly, the 21 patients with HAPS+, 13 patients had Ranson's score less than three, of which four patients were admitted in the general ward for more than 10 days and six patients ultimately required ICU admission. The remaining three patients were discharged within five days after admission in the general ward. The eight patients who were HAPS+ with Ranson's score more than three, all were admitted in the ICU. These patients gradually improved after which they were shifted to the general ward and discharged accordingly. There was no mortality among the patients enrolled in this study.(Figure 2)(Table II)Average length of stay was 4.4 days for HAPS 0 group as compared to 7.2 days for HAPS + group. The data showing the association between the HAPS and on admission Ranson's score with poor prognosis of the patients were statistically significant $(p<0.001)$.

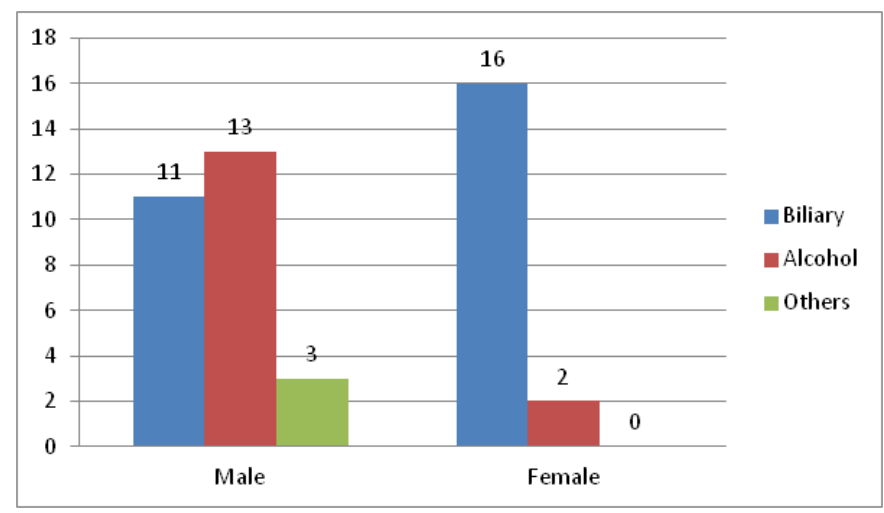

Figure 1: Etiological and sex distribution of AP 


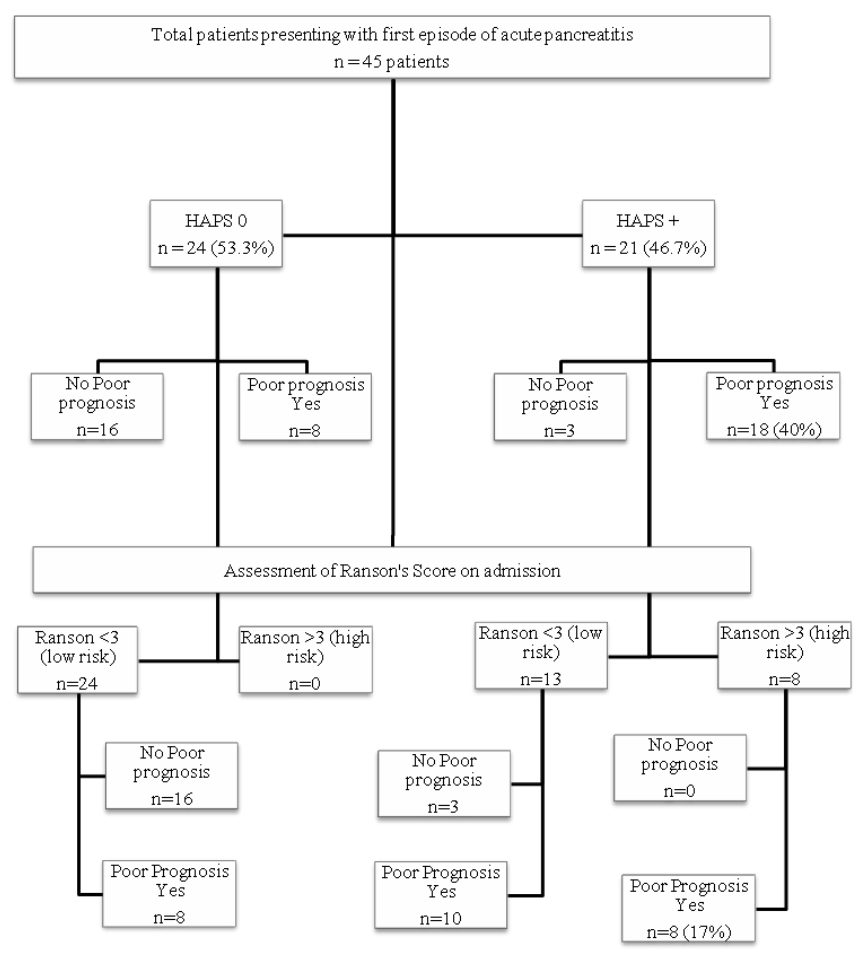

Figure 2: Flow chart of categorization of patients on the basis of Harmless Acute Pancreatitis Score and Ranson's Criteria with Prognosis

$\begin{array}{cccc}\text { Ranson's Score } & \text { HAPS } 0 & \begin{array}{c}\text { Poor Prognosis } \\ \text { Yes }\end{array} & \text { Poor prognosis No } \\ \text { Ranson }<3 \text { (low risk) } & 24 & 8 & 16 \\ \text { Ranson }>3 \text { (high risk) } & 0 & 0 & 0\end{array}$

Table I: Poor prognosis with HAPS 0 and Ranson's Score

\begin{tabular}{|cccc} 
Ranson's Score & HAPS + & $\begin{array}{c}\text { Poor } \\
\text { prognosis Yes }\end{array}$ & $\begin{array}{c}\text { Poor } \\
\text { prognosis No }\end{array}$ \\
Ranson $<3$ (low risk) & 13 & 10 & 3 \\
Ranson $>3$ (high risk) & 8 & 8 & 0
\end{tabular}

Table 2: Poor prognosis with HAPS + and Ranson's Score

\section{DISCUSSION}

AP usually presents with acute persistent upper abdominal pain, nausea and vomiting. ${ }^{11}$ Two most common etiologies for AP are gallstone and alcohol consumption. Gall stone was most common etiology in our study as shown in previous study. ${ }^{12}$ Although, majority of patients runs a mild course, detecting the small portion of patients who will progress to severe disease is important. It is essential to identify these subgroups of patients who will go on to develop adverse outcomes and severe disease early in the course. If appropriate aggressive treatment modality is administered in the early stage of disease then the mortality rate can be lowered. ${ }^{3}$
Syrac AV et al reported that severe pancreatitis was more common in etiology other than billiary. ${ }^{11}$ In our study among forty five patients, in twenty seven patients (60\%) etiology was biliary followed by alcohol consumption in fifteen patients (33\%). Criteria for poor prognosis was present in fourteen (51\%) out of twenty seven patients with biliary etiology, compared to nine $(60 \%)$ out of 15 patients with etiology as alcohol consumption. As in previous study sex was not statistically significantly associated with the severity of AP ( $p$ value more than0.5). ${ }^{13}$ Syrac AV et al reported that HAPS was able to predict poor prognosis in $59 \%$ of patients who did not fulfill HAPS compared to on admission Ranson's score which was able to predict in less than $40 \%$ patients. ${ }^{11}$ In our study predefined poor prognosis were found in twenty one $(46.7 \%)$ of patients among the total 45 patients. Of these severe AP patients, HAPS was able to predict poor prognosis in 18 out of 21 patients (40\%), as compared to Ranson's admission score which was able to predict poor prognosis in eight out of twenty one patients (17.7\%). It was comparable to this study.

Parekh $\mathrm{R}$ et al reported average length of stay in hospital which was 4.3 days for patient with normal HAPS and 6.2 days for patients with abnormal HAPS. ${ }^{14}$ In our study average length of stay in hospital was 4.4 days for patients who were assigned as HAPS 0 compared to 7.2 days for patient who were assigned as HAPS +, which was comparable to this study.

Syrac AV et al reported specificity and positive predictive value of HAPS in determining ICU care was $81 \%$ and $96 \%$ respectively. ${ }^{11}$ Among the 24 patients with HAPS 0 and Ranson's score less than three (low risk), three patients required ICU admission. Similarly, the 21 patients with HAPS+, 13 patients had Ranson's score less than three, of which six patients required ICU admission. The eight patients who were HAPS+ with Ranson's score more than three were admitted in the ICU. Therefore, total 17 patients were admitted in the ICU. The sensitivity of HAPS was $85.71 \%$ and Sensitivity was $66.7 \%$. Although the HAPS score appears to be a promising new stratification scheme for severity in AP, the effect of these hospital-based interventions on the clinical course of their patient cohort should be reported. Until then, patient with predicted harmless disease based on HAPS criteria should not be discharged home from the ED.

\section{LIMITATIONS}

The sample size was small. Similarly, the duration of the study was also a year. Had it been a study of longer duration, larger sample size with more precise and accurate results would have been achieved.

\section{CONCLUSION}

Harmless Acute Pancreatitis Score proved to be a better screening tool compared to on admission Ranson's scoring system to predict the severity of Acute Pancreatitis, which may help predict the prognosis of the patient. 


\section{REFERENCES}

1. Peery AF, Dellon ES, Lund J, et al. Burden of gastrointestinal disease in the United States: 2012 update. Gastroenterology. 2012;143(5):1179-87.

2. Mayerle J, Hlouschek V, Lerch MM. Current management of acute pancreatitis. Nature clinical practice Gastroenterology and Hepatology. 2005;2(10):473-83.

3. Buter A, Imrie CW, Carter CR, Evans S, Mckayn CJ. Dynamic nature of early organ dysfunction determines outcome in acute pancreatitis. British Journal of Surgery. 2002;89(3):29802.

4. Banerjee AK. Audit of fatal pancreatitis. Postgraduate Medical Journal 1995;71(838):472-5.

5. Ranson JH, Rifkind KM, Roses DF, Fink SD, Eng K, Localio SA. Objective early identification of severe acute pancreatitis. American Journal of Gastroenterology. 1974;61(6):443-51.

6. Blamey SL, Imrie CW, O'neill J, Gilmour WH, Carter DC. Prognostic factors in acute pancreatitis. Gut. 1984 ; 25(12):1340-6.

7. Cho JH, Kim TN, Chung HH, Kim KH. Comparison of scoring systems in predicting the severity of acute pancreatitis. World Journal of Gastroenterology. 2015;21(8):2387.

8. Larvin M, Mcmahon M. APACHE-II score for assessment and monitoring of acute pancreatitis. The Lancet. 1989;334(8656):201-5.

9. Wu BU, Johannes RS, Sun X, Tabak Y, Conwell DL, Banks PA. The early prediction of mortality in acute pancreatitis: a large population-based study. Gut. 2008;57(12):1698-703.

10. Wilson C, Heath DI, Imrie CW. Prediction of outcome in acute pancreatitis: a comparative study of APACHE II, clinical assessment and multiple factor scoring systems. British Journal of Surgery. 1990;77(11):1260-4.

11. Sayraç AV, Cete Y, Yiğit Ö, Aydın AG, Sayrac N. Utility of HAPS for predicting prognosis in acute pancreatitis. Ulusal travma ve acil cerrahi dergisi Turkish Journal of Trauma \& Emergency Surgery. 2018;24(4):327-32.

12. Lankisch PG, Apte M, Banks PA. Acute pancreatitis. The Lancet 2015;386:85-96.

13. Uhl W, Warshaw A, Imrie C, et al. IAP Guidelines For the Surgical Management of Acute Pancreatitis. Pancreatology: official journal of the International Association of Pancreatology. 2002;2(6):565-73.

14. Parekh R, Zalawadia A, Dave A, Ambulgekar N, Buran G. Harmless Acute Pancreatitis Score (Haps) As an Initial Predictor of Nonsevere Disease in Acute Pancreatitis. Hospital medicine Dallas Texas. 2011;9:10-13. 